\title{
A Prospective Randomized Study of Prophylactic Irradiation of Tracts in Patients with Malignant Pleural Mesothelioma
}

\author{
Nesreen A. Mosalam, Ahmed A. Nagy \\ Clinical Oncology Department, Faculty of Medicine, Ain Shams University, Cairo, Egypt
}

Background: Procedure tract metastasis (PTM) may complicate pleural procedures in malignant pleural mesothelioma (MPM) patients and cause significant morbidity.

Aim: To evaluate the effectiveness of prophylactic radiotherapy (RTH) in preventing PTM and reducing pain.

Methods: Forty patients with MPM, who had a pleural invasive procedure within the preceding 15 days, were randomized in a 1:1 ratio to receive prophylactic RTH to the procedure site $(21 \mathrm{~Gy}$ in three consecutive daily fractions using $9 \mathrm{MeV})$ vs. no RTH. During a 12-month follow up period, patients were examined monthly for PTM, toxicities and pain at the procedure site.

Results: Patients receiving RTH had lower incidence of PTM than the control group (2/20, 10\% vs. 5/20, 25\%); however, this difference was not statistically significant. The proportion of patients who experienced pain at the pleural procedure site was significantly less in the RTH group compared with the control group $(2 / 20,10 \%$ vs. 12/20, 60\%; $\mathrm{p}=0.001)$. Pain scores were significantly less in the RTH group compared with the control group (mean pain score 1.6 vs. 2.8 , respectively; $\mathrm{p}=0.014)$.

Conclusion: Prophylactic RTH to the pleural procedure site in MPM was not significantly effective in preventing or delaying PTM. However, prophylactic RTH reduced significantly the rate and severity of pain at the procedure site. Future studies may be needed to assess the effect of prophylactic RTH timing and its technique on preventing PTM.

Keywords: Mesothelioma, Radiotherapy, Procedure tract metastasis, Pain.

Corresponding author: Nesreen Mosalam, MD; Clinical Oncology Department, Faculty of Medicine, Ain Shams University, Cairo, Egypt; Email: dr.nesreen2000@gmail.com

Received: 14-June-2017, Revised: 10-July-2017, Accepted: 11-July-2017, Published online: 28-August-2017.

\section{INTRODUCTION}

Malignant pleural mesothelioma (MPM) is a relatively rare aggressive tumor ${ }^{1}$. The incidence of MPM is rising to be almost 2500-3500 cases per year in the United States. Similarly, the incidence is increasing in other parts of the world especially in developing countries like Egypt, where asbestos exposure is high in certain areas with lack of proper protective devices ${ }^{2,3}$. There are many factories using asbestos in Egypt, like the Siegwart factories in Cairo (Shobra El-Khaymah and Helwan districts), and a rising incidence of MPM ${ }^{3,4}$.

Patients with MPM usually undergo pleural procedures during the course of their disease, such as pleural biopsy for tissue diagnosis or drainage of pleural effusion ${ }^{5}$. Pleural effusion occurs in almost all patients with MPM (about 95\%) and dyspnea is the common presenting symptom in many patients. This pleural effusion is usually recurrent and requires frequent pleural tapping or pleurodesis ${ }^{6}$.

Mesothelioma cells have the ability to seed along the pleural procedure tract due to the ability of mesothelioma cells to spread in a sheet-like fashion along the serosal surfaces. Interruption of the tumor sheets allows the malignant cells to spread along the tract created during the pleural procedure from the pleura to the skin resulting in subcutaneous nodules 5 . The procedure tract may be painful and the subcutaneous nodule may be distressing for the patient.
There are few data about the incidence and risk factors for procedure tract metastasis (PTM) and the timing of its development following pleural procedures ${ }^{7}$.

To prevent PTM, prophylactic radiotherapy (RTH) to the sites of pleural procedures in MPM has been investigated in relatively few randomized clinical trials 5, 8-12. Mesothelioma is radiosensitive and RTH has an established role in symptom palliation such as for localized pain. However, RTH is not used with a curative intent due to unacceptable toxicities such as pneumonitis and myocarditis. It has been suggested that prophylactic irradiation of the procedure site may prevent PTM especially with small tumors and that it is more effective than irradiation of already developed metastases ${ }^{13}$.

We conducted this study to evaluate the efficacy of prophylactic RTH in preventing or delaying PTM and improving pain at the site of pleural procedures in MPM patients.

\section{METHODS}

This was an open-label, randomized controlled trial conducted in the Clinical Oncology Department, Faculty of Medicine, Ain Shams University, Cairo, Egypt. The study was approved by the institutional ethics committee and all participants gave an informed consent. 


\section{Participants}

We included patients with MPM who presented to our clinical oncology center from April 2013 till April 2015. Patients who met the following criteria were eligible for inclusion: age $\geq 18$, histologically proven MPM, Eastern Cooperative Oncology Group (ECOG) performance status $\leq 2$, inoperable or unfit for surgery, visible pleural procedure scare at the time of randomization and pleural procedure within two weeks from starting RTH. Patients were excluded in the following conditions: previous RTH to the the pleural procedure site, thoracotomy, other primary malignancy, currently receiving chemotherapy, metastatic disease and sarcomatoid pleural mesothelioma.

\section{Intervention}

The experimental group received prophylactic RTH to the site of pleural procedure while the control group did not receive RTH. Prophylactic RTH was delivered within a maximum of two weeks of the procedure using direct field $9 \mathrm{MeV}$ electron beam in a dose of $21 \mathrm{GY}$ in 3 consecutive daily fractions with $2 \mathrm{~cm}$ margin all around the procedure site if it was a needle site and $3 \mathrm{~cm}$ if it was an intercostal tube site. In case of obese patients or thick chest wall, a skin bolus with $1 \mathrm{~cm}$ thickness was used. The control group did not receive prophylactic RTH but patients who developed PTM during the follow up period received palliative RTH with the same protocol as the experimental group.

\section{Outcomes}

Patients were examined on monthly basis for PTM, RTH toxicities and pain persistence at the procedure site. All patients were followed up for one year from receiving RTH. We assessed acute and late skin toxicities according to the Common Terminology Criteria for Adverse Events (CTCAE) version 4.0 ${ }^{14}$. Pain at the site of pleural procedure or PTM was assessed using the pain score of the National Initiative on Pain Control which is a numeric rating scale ranging from 0 to 10 with higher score indicating more severe pain ${ }^{15}$.

\section{Sample size}

The sample size was calculated using the StatsDirect software (professional version) with a power of $80 \%$ and an alpha level of $5 \%$ to detect a difference in the PTM rate as reported by Bydder et al ${ }^{16}$. The sample size needed for this study was 20 patients in the experimental group and 20 in the control group.

\section{Randomization}

Patients were randomly assigned in a $1: 1$ ratio to the two trial groups. The random sequence was generated based on the day of attendance of the patient. Patients attending on Saturday, Monday, and Wednesday were allocated to the experimental group while those attending on the other days were allocated to the control group until each group reaches a sample size of 20.

\section{Statistical methods}

Statistical analysis was conducted using the Statistical package for Social Science (SPSS 15.0.1 for Windows). Data normality was tested using the Kolmogorov-Smirnov test. Continuous variables were described as mean \pm standard deviation in case of normal distribution and as median and interquartile range in case of non-normal distribution. Categorical data were presented as frequencies and proportions. Outcomes of the two groups were compared using the fisher's exact test. Pooled data from randomized controlled trials were analyzed using the MantelHaenszel method in the Rothman-Boice fixed effect model meta-analysis. An alpha level below 0.05 was considered statistically significant. We followed the CONSORT statement guidelines during the preparation of this manuscript ${ }^{17}$.

\section{RESULTS}

Forty-eight patients were assessed for eligibility. Of them 40 patients were recruited to the two groups (20 patients in each group). The CONSORT flow diagram of the study is shown in figure 1 .

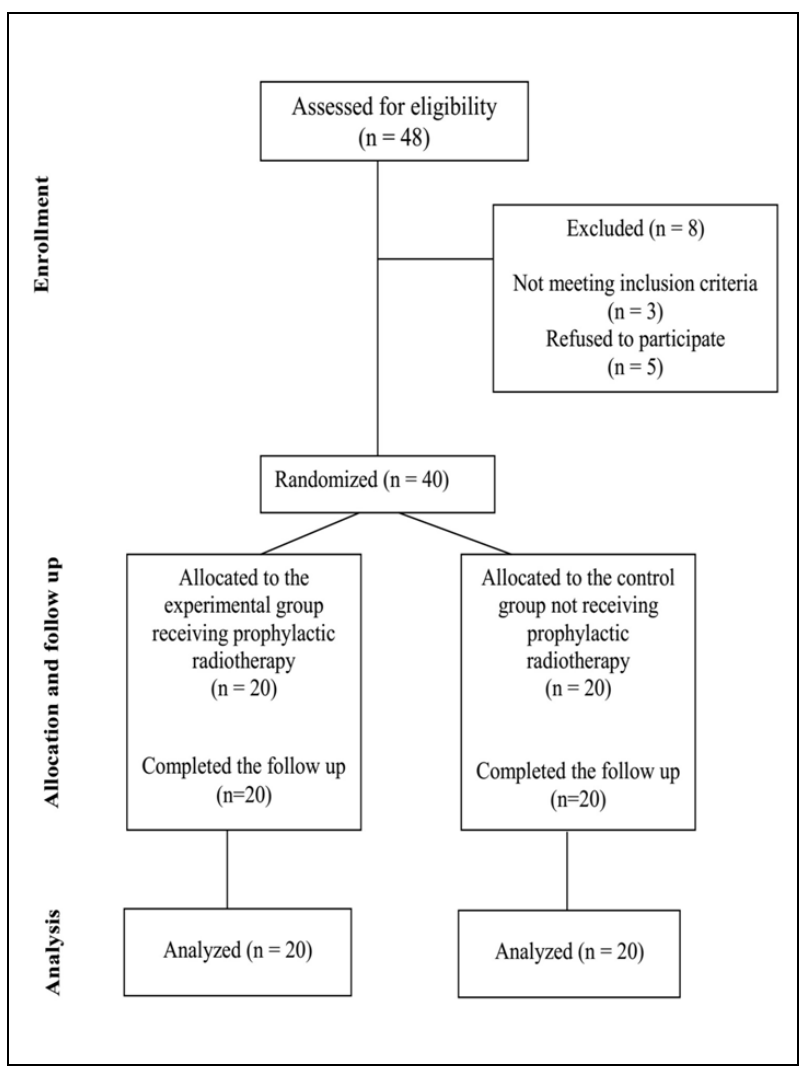

Figure 1. The CONSORT flow diagram of study participants.

The characteristics of the study population of both groups are presented in table 1. There was no statistically significant difference between the two groups in terms of age, gender, performance scores, or pathological type of the tumor.

The proportion of patients who developed PTM within the RTH field was less in the experimental group 
compared to the control group (2/20 vs. 5/20, figure 2$)$. However, this difference was not statistically significant $(p=0.405)$. The development of PTM was not associated with the type of pleural procedure $(\mathrm{p}=0.698)$. The mean time till the development of PTM did not differ significantly between the two groups (RTH group: 7 months vs. control group: 6.3 months, $\mathrm{p}=0.864$ ).

Table 1. Characteristics of patients

\begin{tabular}{|c|c|c|c|}
\hline & $\begin{array}{l}\text { Experimental } \\
\text { Group }(n=20) \\
\end{array}$ & $\begin{array}{l}\text { Control } \\
\text { Group }(n=20) \\
\end{array}$ & p value \\
\hline \multicolumn{4}{|l|}{ Age (years) } \\
\hline Median (IQR) & $52(49-58)$ & $51.5(50-59)$ & 0.384 \\
\hline \multicolumn{4}{|l|}{ Gender } \\
\hline Male & $15(75 \%)$ & $17(85 \%)$ & 0.692 \\
\hline \multicolumn{4}{|l|}{ ECOG* score } \\
\hline 0 & $8(40 \%)$ & $7(35 \%)$ & 0.875 \\
\hline 1 & $10(50 \%)$ & $10(50 \%)$ & \\
\hline 2 & $2(10 \%)$ & $3(15 \%)$ & \\
\hline \multicolumn{4}{|l|}{ Pathological type } \\
\hline Epithelial & $18(90 \%)$ & $19(95 \%)$ & 0.548 \\
\hline Mixed & $2(10 \%)$ & $1(5 \%)$ & \\
\hline \multicolumn{4}{|l|}{$\begin{array}{l}\text { Pleural } \\
\text { procedure }\end{array}$} \\
\hline Needle biopsy & $17(85 \%)$ & $16(80 \%)$ & 0.677 \\
\hline Tubal insertion & $3(15 \%)$ & $4(20 \%)$ & \\
\hline
\end{tabular}

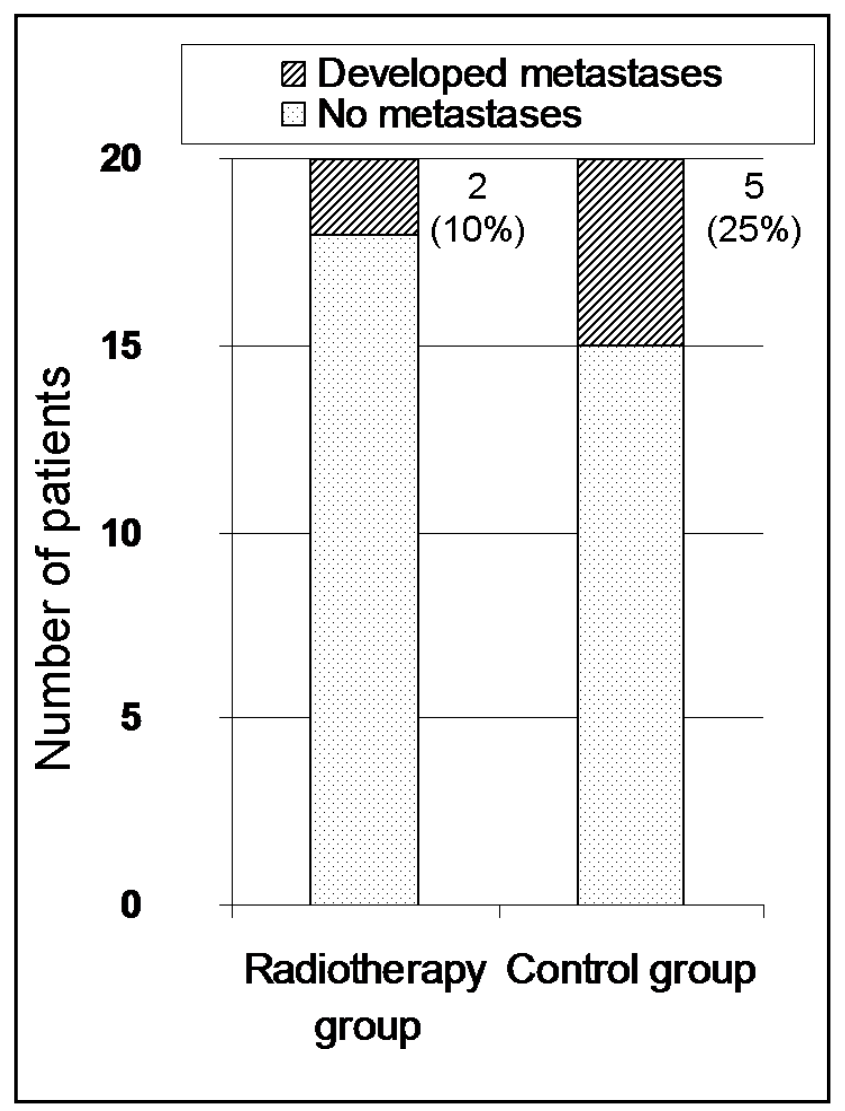

Figure 2: Proportion of patients who developed procedure tract metastasis in both groups
The mean pain score of the RTH group was significantly less than that of the control group (1.6 vs. $2.8, \mathrm{p}=0.014)$. Moreover, the proportion of patients who complained from pain at the pleural procedure site was less in the RTH group compared with the control group $(2 / 20,10 \%$ vs. $12 / 20,60 \% ; p=0.001)$.

In the RTH group, only two (10\%) patients experienced grade one skin erythema.

\section{DISCUSSION}

This randomized control trial showed that prophylactic RTH to the site of pleural procedure might be beneficial for patients with MPM. In this study, the proportion of patients who developed PTM was less in the RTH group than the control group. However, this difference was not statistically significant. Pain score was significantly lower with prophylactic RTH. In terms of safety, no serious adverse events were reported and RTH was well-tolerated.

Other reports in the literature showed lower rate of PTM with prophylactic RTH. In the study conducted by Low et al, none of the 20 MPM patients who received local RTH developed PTM during a follow up period ranging from 1 to 10 months ${ }^{18}$. However, that study lacked a comparator group. Our findings are consistent with that of West et al who found no PTM within the prophylactic RTH area in 37 MPM patients except in two patients $(5 \%)$ who developed invasion at the periphery of previous RTH field ${ }^{19}$.

In our study the mean time till the development of PTM did not differ between the two groups (7 months in the RTH group vs. 6.3 months in the control group), which is similar to that of O'Rourke et al who reported a median time of 2.4 and 6.4 months for the RTH and control groups, respectively, with no significant difference ${ }^{20}$.

Our study showed that patients who received prophylactic RTH had significantly less pain than those in the control group. Moreover, the proportion of patients who complained from pain was significantly less in the RTH group. This highlights the effectiveness of RTH therapy in reducing pain. We do not have an explanation for the discrepancy in the significance of PTM prevention and pain reduction.

Three randomized controlled trials including relatively small sample sizes investigated the role of prophylactic RTH in reducing PTM 16, 20, 21. Our findings are consistent with that of Boutin et al ${ }^{21}$ and Bydder et al ${ }^{16}$ but not with that of O' Rourke et al ${ }^{20}$. In the study of Boutin et al, forty patients were randomized to the RTH group $(n=20)$ or control group $(n=20)^{21}$. No patients $(0 \%)$ in the RTH group developed PTM but 8 (40\%) patients in the control group developed PTM. Our study differs from that of Boutin et al in the types of pleural procedures included. In our study, only pleural biopsy and tubal insertion were included because thoracoscopy was not performed in our center during the study period. Bydder et al ${ }^{16}$ randomized 43 MPM patients to receive a $10-\mathrm{Gy}$ single dose of prophylactic RTH vs. no RTH. The proportion of patients who developed PTM in the RTH group was less than the 


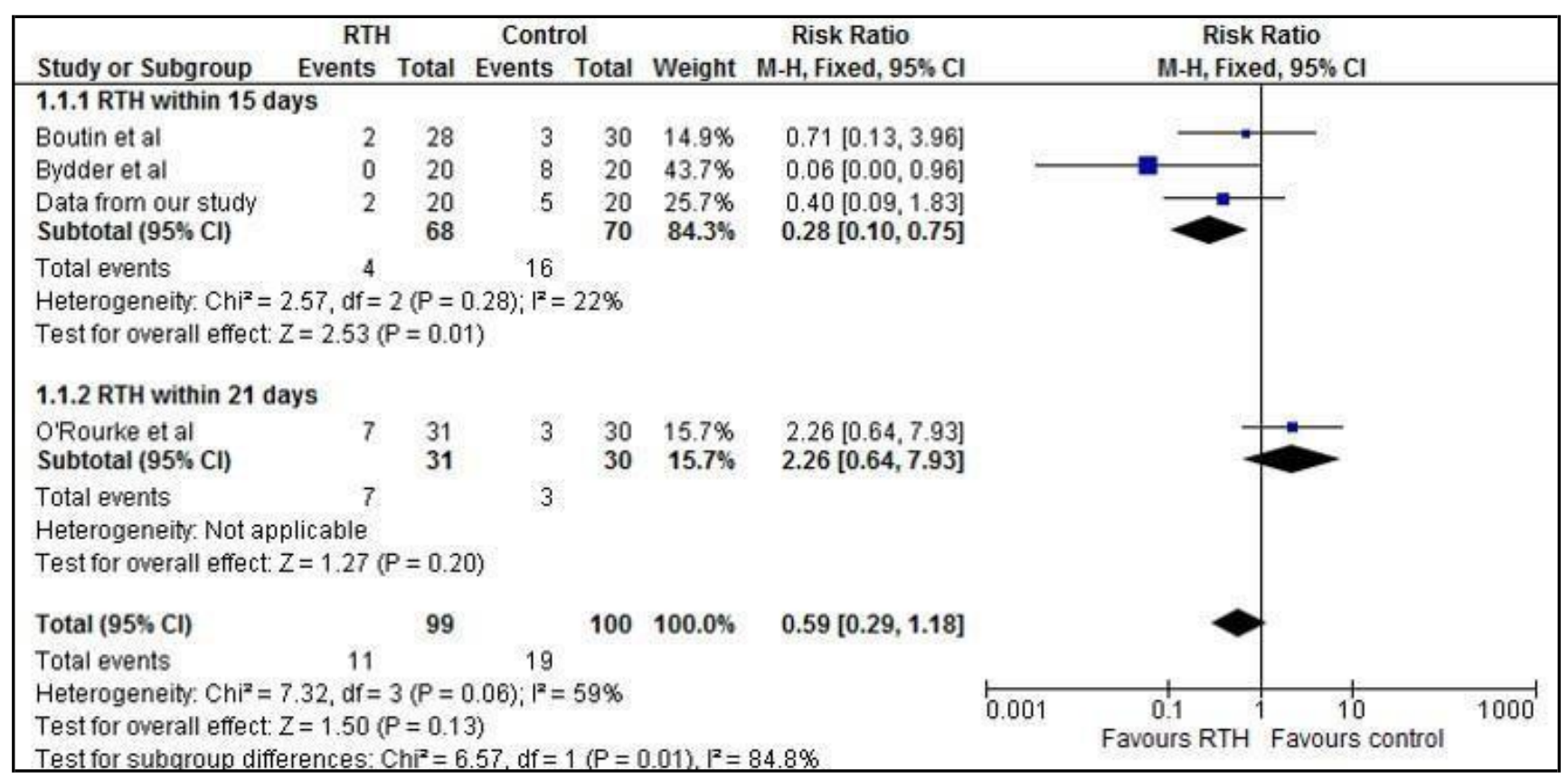

\section{Figure 3: Forest plot of the efficacy of prophylactic radiotherapy (RTH) in reducing procedure tract metastasis.}

control group (7\% vs. $10 \%$, respectively). However, this difference was not significant. It should not escape our notice that they used a different RTH regimen (10-Gy single fraction using $9 \mathrm{MeV}$ ).

In the third trial done by $\mathrm{O}^{\prime}$ Rourke et al, 61 patients were randomized to prophylactic RTH vs. no RTH ${ }^{20}$. The proportion of PTM in the RTH group $(7 / 31,23 \%)$ was higher than that in the control group $(3 / 30,10 \%)$. These results are contradictory to our findings and those of the other two randomized controlled trials. This may be explained by the fact that O' Rourke et al ${ }^{20}$ delivered prophylactic RTH within 21 days from the pleural procedure, while it was delivered within 15 days in our trial and in the other two trials ${ }^{16}$, ${ }^{21}$. This suggests that the timing of prophylactic RTH is a contributing factor to its efficacy.

The result of pooled analysis of the abovementioned three randomized controlled trials in addition to ours is not in favor of using prophylactic RTH to prevent PTM (RR 0.59, 95\% CI: 0.29-1.18, p=0.13).

There was significant heterogeneity in these data which was resolved by subgroup analysis to RTH within 15 days vs. RTH within 21 days (figure 3). Prophylactic RTH was significantly superior to no RTH in reducing PTM in the subgroup of studies where RTH was given within 15 days. This difference between the two subgroups (RTH within 15 days vs. RTH within 21 days) was statistically significant $(\mathrm{p}=0.01)$.

According to the recommendation of the European Society of Medical Oncology (ESMO) 2015 for the diagnosis, treatment, and follow up of MPM, evidence about the efficacy of prophylactic RTH in preventing PTM is controversial and it should not be routinely applied $^{22}$.

Based on the results of our randomized controlled trial and those of previous trials, we believe that prophylactic RTH to the pleural procedure site might be effective in preventing PTM. However, the current evidence is not sufficient to confirm its efficacy. Future studies should investigate the effect of RTH timing (within 15 days vs. 21 days) and the RTH technique (10 Gy in single fraction vs. 21 Gy in three fractions) on the efficacy of prophylactic RTH to prevent PTM. Additionally, the effect of prophylactic RTH on pain and quality of life of MPM patients should be explored.

\section{Conclusion}

Data from our randomized controlled trial showed that prophylactic RTH to the pleural procedure site in MPM patients was not significantly effective in preventing or delaying PTM. However, our study shows that prophylactic RTH is effective in reducing pain at the procedure site.

\section{REFERENCES}

1. West SD, Lee YC. Management of Malignant Pleural Mesothelioma. Clin Chest Med. 2006; 27(2):335-354.

2. Robinson BW, Musk AW, Lake RA. Malignant Mesothelioma. Lancet. 2005; 366(9483):397-408.

3. Gaafar RM, Eldin NH. Epidemic of Mesothelioma in Egypt. Lung Cancer. 2005; 49 (Suppl 1): S17-20.

4. Akl Y, Kaddah S, Abdelhafeez A, Salah R, Lotayef M. Epidemiology of Mesothelioma in Egypt. A Ten-Year (1998-2007) Multicentre Study. Arch Med Sci. 2010; 6(6): 926-931.

5. Arnold DT, Clive AO. Prophylactic Radiotherapy for Procedure Tract Metastases in Mesothelioma: A Review. Curr Opin Pulm Med. 2017; 23(4): 357-364.

6. Bayman N, Ardron D, Ashcroft L, et al. Protocol for PIT: A Phase III Trial of Prophylactic Irradiation of Tracts in Patients with Malignant Pleural Mesothelioma Following Invasive Chest Wall Procedure. BMJ Open. 2016; 6(1): e010589.

7. Davies HE, Musk AW, Lee YC. Prophylactic Radiotherapy for Pleural Puncture Sites in Mesothelioma: The Controversy Continues. Curr Opin Pulm Med. 2008; 14(4): 326-330. 
8. American Thoracic Society. Management of Malignant Pleural Effusions. Am J Respir Crit Care Med. 2000; 162(5): 1987-2001.

9. De Ruysscher D, Slotman B. Treatment of Procedure Sites of Malignant Pleural Mesothelioma with Radiotherapy: A Dutch-Belgian Survey. Radiother Oncol. 2003; 68(3):299-302.

10. British Thoracic Society Standards of Care Committee. BTS statement on malignant mesothelioma in the UK, 2007. Thorax. 2007; 62 (Suppl 2): ii1-ii19.

11. Scherpereel A; French Speaking Society for Chest Medicine (SPLF) Experts Group. Guidelines of the French Speaking Society for Chest Medicine for Management of Malignant Pleural Mesothelioma. Respir Med. 2007; 101(6):1265-1276.

12. Robinson BW, Lake RA. Advances in Malignant Mesothelioma. N Engl J Med. 2005; 353(15):15911603.

13. Waite K, Gilligan D. The Role of Radiotherapy in the Treatment of Malignant Pleural Mesothelioma. Clin Oncol (R Coll Radiol). 2007; 19(3): 182-187.

14. US Department of Health and Human Services. National Cancer Institute. Common Terminology Criteria for Adverse Events (CTCAE) Version 4.0. National Institutes of Health, National Cancer Institute; 2009. Available from: http://evs.nci.nih.gov/ftp/CTCAE V4.03.

15. McCaffery M, Pasero C. Pain: Clinical manual. St Louis, MO: Mosby, 1999.
16. Bydder S, Phillips M, Joseph DJ, et al. A Randomised Trial of Single-Dose Radiotherapy to Prevent Procedure Tract Metastasis by Malignant Mesothelioma. $\mathrm{Br} \mathrm{J}$ Cancer. 2004; 91(1):9-10.

17. Moher D, Hopewell S, Schulz KF, et al. CONSORT 2010 Explanation and Elaboration: Updated Guidelines for Reporting Parallel Group Randomised Trials. BMJ 340:c869.

18. Low EM, Khoury GG, Matthews AW, Neville E. Prevention of Tumour Seeding Following Thoracoscopy in Mesothelioma by Prophylactic Radiotherapy. Clin Oncol (R Coll Radiol). 1995; 7(5): 317-318.

19. West SD, Foord T, Davies RJ. Needle-Track Metastases and Prophylactic Radiotherapy for Mesothelioma. Respir Med. 2006; 100(6): 1037-1040.

20. O'Rourke N, Garcia JC, Paul J, Lawless C, McMenernin R, Hill J. A Randomised Controlled Trial of Procedure Site Radiotherapy in Malignant Pleural Mesothelioma. Radiother Oncol. 2007; 84(1): 18-22.

21. Boutin C, Rey F, Viallat JR. Prevention of Malignant Seeding after Invasive Diagnostic Procedures in Patients with Pleural Mesothelioma: A Randomized Trial of Local Radiotherapy. Chest. 1995; 108(3): 754-758.

22. Baas P, Fennell D, Kerr KM, Van Schil PE, Haas RL, Peters S; ESMO Guidelines Committee. Malignant Pleural Mesothelioma: ESMO Clinical Practice Guidelines for Diagnosis, Treatment and Follow-Up. Ann Oncol. 2015; 26 (Suppl 5): v31-39. 